Artículo Especial

\title{
Sistemas de contención de órganos en movimiento: Radioterapia Adaptada a la Respiración (RAR)\#
}

\author{
M. E. Castellanos, T. Lacornerie, B. Prevost, X. Mirabel, E. Lartigau
}

\section{Introducción}

La anatomía de los pacientes y su posición durante un tratamiento de radioterapia usualmente difieren de los usados para la planificación del mismo. Esto se debe principalmente al movimiento del paciente, a su posicionamiento incorrecto y al movimiento de sus órganos. En consecuencia, en general, las distribuciones de dosis administradas a los pacientes son diferentes a las planificadas. Con el objetivo de asegurar la presencia del tumor en todo momento en el volumen irradiado los radioterapeutas están obligados a recurrir a haces de dimensiones más grandes que las del volumen blanco y a irradiar una porción más importante de tejidos sanos. En efecto, el movimiento del volumen tumoral es generalmente tenido en cuenta usando márgenes que lo rodean, para la definición de los cuales las recomendaciones internacionales más importantes han sido propuestas por la Comisión Internacional de Unidades y Medidas de Radiación (Reportes ICRU 50 e ICRU 62). A falta de datos precisos, los radioterapeutas han adoptado márgenes empíricos de 1,5 a $2,0 \mathrm{~cm}$ obtenidos de experiencia de la radioterapia convencional.

En el caso particular de los tumores de tórax y abdomen en radioterapia convencional se acepta, además, que el paciente respire libremente durante los estudios tomográficos para planificación y durante el

Centro Regional de Lucha contra el Cáncer Centro Oscar Lambret

Lille (Francia)

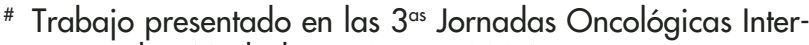
nacionales, Madrid 17-19 junio 2004. tratamiento mismo, bajo la presunción de que los efectos de la respiración se promedian. El desarrollo de la radioterapia conformal con o sin modulación de intensidad ha convertido ésta en una situcción poco aceptable, puesto que el enfoque conformal está basado en una definición más precisa de la posición anatómica de los volúmenes blancos tumorales, con el fin de reducir las dimensiones de los haces y de esa manera proteger mejor los tejidos sanos, con miras al escalamiento de la dosis (Hanley et al, 1999).

\section{Influencia de la respiración}

Durante los últimos cinco años la comunidad internacional ha dedicado especial atención a la búsqueda de mecanismos para reducir esos movimientos y en consecuencia los márgenes, lo cual se ha logrado muy bien en el tratamiento de tumores de cabeza y cuello y de pelvis, en particular, mediante el uso rutinario de inmovilizadores ya ampliamente conocidos. Los esfuerzos se orientan ahora hacia la disminución de la influencia de la respiración en el tratamiento de tumores abdominales y torácicos. Diferentes aspectos están siendo estudiados: la influencia de la respiración en la adquisición de las imágenes tomográficas, las consecuencias de esa influencia en el cálculo de la distribución de la dosis y de las unidades monitoras y la influencia de la respiración en la administración del tratamiento.

Los avances recientes en la planificación y administración de tratamientos en radioterapia hacen posible realizar cálculos y administrar distribuciones de dosis que se adaptan (conforman) muy bien a los volúmenes blancos. Sin embargo, la predicción de la dosis administrada por un arreglo complejo de haces, está rela- 
cionada con una descripción exacta del paciente y errores en el modelo del paciente usado en la planificación del tratamiento pueden llevar a estimaciones erróneas de la posición de órganos, de la cobertura dosimétrica de las estructuras críticas, dentro o cerca de las regiones de altas dosis, así como de las profundidades. Aunque, gracias a los cortos tiempos de muestreo en la tomografía computarizada $(<2 \mathrm{~s} /$ corte) y a los períodos más largos de ventilación, la respiración libre puede no afectar significativamente la resolución de la imagen. Ella introduce principalmente tres tipos de incertidumbres: incertidumbres geométricas en los datos de la tomografía, diferencias en la cantidad de tejido denso atravesado por el haz, lo cual altera el recorrido de la radiación hasta el centro del tumor y finalmente incertidumbres en la estimación de los volúmenes de las estructuras críticas, lo cual puede llevar a conclusiones erróneas en la evalucción del plan.

La aparición de sistemas helicoidales rápidos de tomografía, a comienzos de los años 90 , trajo consigo la posibilidad de obtener volúmenes anatómicos en un solo tiempo respiratorio y consecuentemente la posibilidad de comparar adquisiciones hechas en diferentes tiempos del ciclo respiratorio, para medir la amplitud de los movimientos torácicos. Esto permite, a su vez, determinar de manera más objetiva los márgenes que deben ser trazados alrededor de volúmenes tumorales visibles en las imágenes tomográficas. Sin embargo, la reducción de márgenes alrededor de los volúmenes blancos impone también seguir los movimientos inducidos por la respiración con el fin de administrar la radiación en un momento preciso del ciclo respiratorio.

Esa comparación puede ser realizada mediante estudios de fluoroscopia o de tomografía axial. El paciente se somete a una sesión estandarizada de entrenamiento en la cual se le solicita contener la respiración en cuatro puntos del ciclo respiratorio mientras se le mantiene en visulización flucroscópica, por ejemplo; las imágenes obtenidas son usadas para registrar y medir el movimiento del diafragma y del tumor. Estudios de este tipo demostraron que en inspiración o expiración máxima hay una mejor reproducibilidad de la posición, con una desviación estándar promedio de $2.6 \mathrm{~mm}$ en la posición del diafragma y que la variación de la posición del diafragma de una sesión a otra es inferior a $5 \mathrm{~mm}$. Se demostró también que incluso una técnica de auto-bloqueo de la respiración (Kim et al, 2001) reduce el movimiento diafragmático en promedio en $12 \mathrm{~mm}$, en comparación con la respiración libre.

Balter et al (1996), Giroud et al (2001) y Langen et al (2001), entre otros, concluyeron en su estudios que el movimiento de las estructuras torácicas durante las fases extremas de la respiración son considerables, particularmente en regiones vecinas del diafragma. Esto puede conllevar variaciones superiores a $1,5 \mathrm{~cm}$ en la longitud del recorrido ponderado por la densidad del tejido, por ejemplo en haces usados para tratar tumores torácicos y abdominales, cuando se comparan los planes de tratamiento calculados en estudios tomográficos tomados en inspiración y expiración, conservando las mismas coordenadas del isocentro. Esos cambios pueden a su vez llevar a una ponderación inadecuada de los haces, siendo la influencia en la distribución de la dosis más importante para haces de baja energía. En el caso de haces de alta energía usados para tratar tumores a profundidades entre 10 y 15 $\mathrm{cm}$, esos cambios en el recorrido pueden llevar a diferencias de 2-3\% en parámetros dosimétricos básicos como la Relación Tejido-Maniquí (TPR). Esos estudios comprobaron también movimientos de órcanos como el riñón y el hígado de hasta $2 \mathrm{~cm}$ y diferencias de hasta $12 \%$ en el volumen de órganos entre el momento de la inspiración y el momento de la expiración.

\section{Bloqueo respiratorio}

Se han propuesto diferentes estrategias para reducir los errores asociados a los movimientos respiratorios en la planificación y administración del tratamiento. La aproximación más simple consiste en minimizar el movimiento mismo y la forma más simple de hacerlo es bloqueando la respiración. Esto se puede hacer en forma pasiva (Kim et al, 2001; Hanley et al, 1999; Mah et al, 2000) o en forma activa (Wong et al, 1999). El bloqueo, habitualmente en inspiración, de la respiración del paciente se realiza durante la adquisición de las imágenes tomográficas y durante la irradiación del paciente.

Entre las estrategias pasivas la más conocida es la Técnica del Bloqueo de la Respiración en Inspiración Profunda (Deep Inspiration Breath Hold Technique, $D I B H$, Haniey et al, 1999), con dos características importantes: inspiración profunda, lo cual reduce la densidad del pulmón y bloqueo de la respiración, lo cual inmovilizó los tumores de pulmón. Diferentes métodos de DIBH han sido propuestos. El más conocido es el método de monitoreo por espirómetro desarrollado en el Memorial Sloan-Kettering Center. El paciente comienza respirando normalmente para luego hacer una inspiración profunda lenta seguida por una expiración profunda lenta y una inspiración profunda máxima, después de lo cual bloquea la respiración. Este es el sistema aplicado en el Centro Oscar Lambret, de Lille. Otros centros (Kim et al, 2001; Barnes et al, 2001) proponen una radioterapia autoadaptada en la cual el 


\section{E. Castellanos y cols.}

paciente controla un interruptor y el tecnólogo inicia la irradiación cuando el paciente juzga que ha logrado el nivel correcto de bloqueo de la respiración.

En una de las técnicas activas propuesta, el Control Activo de la Respiración (Active Breathing Control, $A B C$, Wong et al, 1999) el bloqueo se realiza mediante un sistema consistente en dos pares de monitores de flujo y válvula, una de las cuales controla la inspiración y otra la expiración del paciente. La señal obtenida es procesada de manera que indique los cambios de volumen del pulmón en tiempo real. Una vez que el patrón de respiración del paciente se vuelve estable el operador activa el sistema $A B C$ en una fase preseleccionada del ciclo respiratorio, se cierran entonces ambas válvulas produciendo un bloqueo controlado de la respiración y la radioterapia solamente es administrada durante el bloqueo.

Diferentes centros han reportado aplicar estos sistemas pasivos y activos, principalmente en estudios de factibilidad y en investigación; ellos mismos han concluido que los sistemas de radioterapia adaptada a la respiración requieren una participación importante de personal (vigilancia) y de los pacientes mismos (esfuerzos respiratorio a veces difíciles), aspectos a tener en cuenta en la organización de un servicio de radioterapia, aún si esta participación activa de los pacientes puede representar una ventaja práctica.

Las ventajas dosimétricas reportadas de estos procedimientos de bloqueo respiratorio han sido principalmente la constatación de la posibilidad de escalamiento de dosis, por cuanto se irradia menos tejido sano. En particular, se demostró que la técnica de DIBH puede reducir el volumen de pulmón que recibe más de 25 Gy en $30 \%$, en comparación con los planes de tratamiento en respiración libre, en casos de tratamiento de tumores de pulmón.

\section{Respiración libre y radioterapia adaptada}

Contrariamente al bloqueo respiratorio, otra estrategia consiste en seguir en tiempo real el ritmo ventilatorio espontáneo del paciente y en adaptar la adquisición de las imágenes y la irradiación a la respiración libre del paciente. La adquisición de la imagen y la irradiación se realiza siempre en un nivel, siempre idéntico, del ciclo respiratorio (Respiratory Gating) que es determinado por captores mecánicos, luminosos o espirométricos. Se usan entonces monitores de respiración externos al paciente para deducir la posición de órganos internos.

Este tipo de sistemas han sido utilizados desde hace más de una década (Ohara et al, 1989) en tratamientos con partículas pesadas y en los últimos años en instalaciones con aceleradores lineales para radioterapia fotónica (Kubo et al, 1996). Un sistema disponible comercialmente es el llamado Real-Time Position Management, RP de Varian el cual consiste en un bloque de plástico colocado sobre la parte alta del abdomen del paciente, sobre el cual se encuentran dos reflectores. Esos reflectores reenvían una luz infrarroja sobre una cámara unida a un computador. El movimiento de los reflectores durante la respiración es analizado por un programa que pilotea la adquisición de la imagen o el inicio de la irradiación.

Antes de cada sesión el técnico coloca el sistema en modo de "rastreo" (tracking) durante unos cuantos ciclos respiratorios con el fin de determinar las posiciones inferior y superior y esperar la estabilización de la respiración, para luego pasar a modo de registro durante el cual se irradia el paciente solamente en períodos previamente establecidos.

Berson et al (2002) evaluaron la RPM en 136 pacientes con patologías tumorales muy diversas (pulmón, hígado, seno, mediastino). De la experiencia en los 108 pacientes irradiados, se confirmó la factibilidad de la aplicoción del sistema: 97 de los 108 pacientes pudieron ser tratados completamente con el dispositivo. El tiempo total de una sesión de tratamiento fue 4 veces superior en promedio, en comparación con la radioterapia conformal estándar. Ford et al (2002) utilizaron esta técnica sobre 8 pacientes tratados por tumores pulmonares o hepáticos. También en este estudio la tolerancia fue excelente: los movimientos residuales medidos por fluoroscopia o imágenes portales fueron evaluados respectivamente en 2,6 $1,7 \mathrm{~mm}$ y $2,8 \pm 1,0 \mathrm{~mm}$. Wagman et al (2003) evaluaron esta técnica para 10 pacientes que presentaban un carcinoma hepatocelular. En esos 10 pacientes, solamente 8 fueron realmente irradiados con RPM, lo cual representa 177 irradiaciones a justadas a la respiración. Los movimientos en el sentido craneocaudal del diafragma fueron reducidos en promedio de 22,7 $\pm 7,4 \mathrm{~mm}$ en respiración libre a 5,1 $\pm 2,1 \mathrm{~mm}$ con la sincronización respiratoria. La diferencia en la posición del diafragma entre las DRR y las imágenes portales fue en promedio de 2,3 $\mathrm{mm}$. La reducción de los movimientos respiratorios del hígado permitió disminuir los márgenes de seguridad en $1 \mathrm{~cm}(1 \mathrm{~cm}$ de margen en total en lugar de 2 usados inicialmente), y en consecuencia, autorizar un aumento de la dosis total de 7 a $27 \%$ según el paciente (valor medio: $21,3 \%)$. Sobre el plano práctico, se estableció que la irradiación con RPM prolonga la duración de la sesión en 10 minutos. 


\section{Otras estrategias}

Otra posibilidad aparentemente mejor, pero más difícil de realizar consistiría en permitir al paciente respirar libremente, mientras un sistema de control y de rastreo (tracking) monitorea la posición del tumor y adapta continuamente la alineación del haz de radiación para seguir el movimiento del blanco (Mackle et al, 2003; Remouchamps et al, 2003; Murply et al, 2004). Se trata de una radioterapia conformal guiada por imágenes, la cual representa actualmente un área activa de investigación.

Todas esas estrategias de compensación de la respiración que identifican la posición del tumor a partir de señales externas de la respiración requieren un conocimiento tridimensional de la trayectoria del movimiento del tumor así como la capacidad de detectar y aduptarse a las características de los cambios transitorios o continuos de los movimientos respiratorios durante el tratamiento.

\section{Referencias bibliográficas}

Balter JM, Ten Haken RK, Lawrence TS, Lam KL, RoLertson JM. Uncertuinties in CT-based radiation therapy treatment planning associated with patient breatRing. Int J Radiat Oncol Biol Phys 1996; 36:167-74.

Barnes EA, Murray BR, Robinson DM, Underwood U, Hanson J, Roa WH. Dosimetric evaluation of lung tumor immobilization using breath hold at deep inspiration. Int J Radiat Oncol Biol Phys 2001; 50(4):1091-8.

Berson AM, Emery R, Ng T, Rodriguez L, SangRavi S. Clinical experience using respiratory gated radiation therapy for tumors in the chest, upper aLdomen, and breast. In: Press P, editor. ASTRO 2002; New Orleans; 2002. p. 1064.

Dawson LA, Brock KK, Kazanjian S, Fitch D McGinn CJ Lawrence $T S$, et al. The reproducibilit, $v$ of órgan position using active breatRing control $(A B C)$ during liver radiotherapy. IntJ Radiat Oncol Biol Phys 2001; 51(5):1410-21.

Ekberg L, Holmberg $O$, WiHgren L, BjelLengren $G$, Landberg T. What margins should be adUed to the clinical target volume in radiotherapy treatment planning for lung cancer? Radiother Oncol 1998; 48:71-7.

Engelsman $M$, Damen EM, De Jaeger $K$, van Ingen KM, Mijnheer BJ. The effect of breathing and set-up errors on the cumulative dose to a lung tumor. Radiother Oncol 2001; 60(1):95-105.

Ford EC, Mageras GS, Yorke E, Rosenzweig KE, Wagman R Ling CC. Evaluation of respiratory movement during gated radiotherapy using film and electronic portal imaging. Int J Radiat Oncol Biol Phys 2002; 52(2):522-31.

Giroud P, De Rycke Y, Dubroy B, Helfre S, Voican D, Guo L, et al. Conformal radiolLerapy (CRT) planning for lung cancer: analysis of intrathoracic organ motion during extreme pRases of breathing. Int J Radiat Oncol Biol Phys 2001; 51(4):1081-92.

Hanley J, Delois MM, Mah D, Mageras GS, Raben A, Rosenzweig K, et al. Deep inspiration breath-hold tecEnique for lung tumors: the potential value of target immobilization and reduced lung density in dose escalation. Int J Radiat Oncol Biol Phys 1999; 45(3):603-11.

International Commission on Radiation Units and Measurements. ICRU Report 62: Prescribing, Recoring, and Reporting Photon Beam Therapy (Supplement to ICRU Report 50); 1999.

Kim DJ, Murroy BR, Halperin R, Roa WH. Held-breath selfgating tecEnique for radiotherapy of non-small-cell lung cancer: a feasibility study. Int $\mathrm{J}$ Radiat Oncol Biol Phys 2001; 49(1):43-9.

Kubo HD, Hill BC. Respiration gated radiotherapy treatment: a technical study. Phys Med Bial 1996; $41(1): 83$.

Langen KM, Jones DT. Organ motion and its management. Int J Radiat Oncol Biol Phys 2001; 50(1):265-78.

Mageras G. Respiration correlated CT techniques for gated treatment of lung cancer. Radiother Oncol 2002; 64 (Suppl 1 ):S75.

Mageras GS, Yorke E, Rosenzweig K, Braban L, Keatley E, Ford $E$, et al. Fluoroscopic evaluation of diapEragmatic motion reduction with a respiratory gated radiotherapy system. J Appl Clin Med Phys 2001; 2(4):191-200.

Mah D, Haniey J, Rosenzweig KE, YorLe E, Braban L, Ling $C C$, et al. Technical aspects of the deep inspiration breath-hold technique in the treatment of thoracic cancer. Int J Radiat Oncol Biol Phys 2000; 48(4): 1 175-85.

Mackie TR, Kapatoes J, Ruchala K. Image guidence for precise conformal radiollerapy. Int J Radiat Oncol Biol Phys 2003; 56:89-105.

Murphy MJ, TracLing Moving Organs in Real Time. Seminars in Radiation Oncology 2004; 14(1):91-100.

Ozhasoglu C, Murphy MJ. Issues in respiratory mation compensation during external-beam radiotherapy. Int J Radiat Oncol Biol Phys 2002; 52(5):1389-99.

Remouchamps VM, Vicini FA, Sharpe MB, Kestin LL, Martinez AA, Wong JW. Significant reductions in heart and lung doses using deep inspiration breath hold with active breathing control and intensity-modulated radiation therapy for patients treated with locoreglonal breast irradiation. Int J Radiat Oncol Biol Phys 2003; 55(2):392-406.

Sixel KE, Aznar MC, Ung YC. Deep inspiration breath hold to reduce irradiated heart volume in breast cancer patients. Int J Radiat Oncol Biol Phys 2001; 49(1):199-204.

Vedam SS, Keall PJ, Kini VR, Moban R. Determining parameters for respiration-gated radiotherapy. Med Phys $2001 ; 28(10): 2139-46$

Wagman R, Yorke E, Ford E, Giroud P, Mageras G, Minsky $B$, et al. Respiratory gating for liver tumors: use in dose escalation. Int J Radiat Oncol Biol Phys 2003; 55(3):65968. 


\section{E. Castellanos y cols.}

Willett CG, Linggood RM, Stracher MA, Goitein M, DoppLe $K$, Kushner DC, et al. The effect of the respiratory cycle on mediastinal and lung dimensions in Hodgkin's disease. Implications for radiotherapy gated to respiration. Cancer 1 987; 60(6): 1232-7.

Wong JW, Sharpe MB, Jaffroy DA, Kini VR, RoLertson JM, Stromberg JS, et al. The use of active breatRing control
$(A B C)$ to reduce margin for breatling motion. Int J Radiat Oncol Biol Phys 1999; 44(4):911-9.

Yorke ED, Wang L, Rosenzweig KE, Mah D, Paoli JB, Chui CS. Evaluation of deep inspiration breath-hold lung treatment plans wilh Monte Carlo dose calculation. Int J Radiat Oncol Biol Phys 2002; 53(4): 1058-70. 